\title{
Proposta de Solução para Aplicação de Internet das Coisas nas Redes Elétricas Inteligentes Brasileiras Considerando as Limitações das Normas Vigentes*
}

\author{
Efrem E. O. Lousada ${ }^{1}$, Raquel A. F. Mini ${ }^{2}$ \\ ${ }^{1}$ Instituto de Informática - Instituto Federal do Norte de Minas Gerais (IFNMG) \\ Pirapora - MG - Brasil \\ ${ }^{2}$ Departamento de Ciência da Computação \\ Pontifícia Universidade Católica de Minas Gerais - Belo Horizonte - Brasil \\ efrem.lousada@ifnmg.edu.br, raquelmini@pucminas.br
}

\begin{abstract}
Advances in the areas of embedded systems, computing and networking have created thousands of heterogeneous devices. Electronic electricity meters are examples of these devices that interconnected can be part of a larger network called Smart Grids. Applying the concept of Internet of Things in these networks, meters should provide the collected information to all system users through servers in the cloud. This work specifies and implements a Brazilian smart meters network, considering the limitations imposed by Brazilian standards, and proposes a technique for calculating the duty cycle of the network dynamically in order to maximize the network lifetime. Experimental results show that the proposed solution, using dynamic duty cycle, increases significantly the network lifetime.
\end{abstract}

Resumo. Avanços nas áreas de sistemas embarcados, computação e redes têm criado milhares de dispositivos heterogêneos. Os medidores eletrônicos de energia elétrica são exemplos desses dispositivos que interconectados podem fazer parte de uma rede maior chamada de redes elétricas inteligentes. A partir da aplicação de internet das coisas nessas redes, os medidores passam a disponibilizar as informações coletadas a todos os usuários do sistema através de servidores na nuvem. Este trabalho especifica e implementa uma rede de medidores inteligentes brasileiros, considerando as limitações impostas pelas normas brasileira, além de propor uma técnica para cálculo do ciclo de trabalho da rede de forma dinâmica com o objetivo de maximizar o tempo de vida. Resultados experimentais mostram que a proposta de utilização de ciclo de trabalho dinâmico aumenta significativamente o tempo de vida da rede.

\section{Introdução}

$\mathrm{O}$ crescente desenvolvimento das aplicações embarcadas tem proporcionado inteligência computacional a diversos tipos de dispositivos. Os medidores de energia elétrica, por exemplo, são microprocessados e deixaram de registrar somente o consumo de energia do consumidor. A possibilidade de comunicação remota com os medidores de energia

\footnotetext{
${ }^{*}$ Este trabalho foi parcialmente financiado pela empresa Nansen S/A Intrumentos de Precisão, CNPq e Fapemig.
} 
elétrica e a criação de redes entre eles foram os primeiros passos para a criação das redes elétricas inteligentes ou smartgrids. Utilizando o conceito de geração e compartilhamento de informações relevantes para a sociedade, a partir de aparelhos computacionais interligados, chamado de Internet das Coisas (IoT - Internet of Things), a aplicabilidade das redes elétricas inteligentes pode ser expandida. As informações e serviços gerados pelos medidores eletrônicos de energia elétrica podem ser compartilhados com outros dispositivos ou pessoas interessadas nas informações [Bello and Zeadally 2014].

O grande desafio no desenvolvimento de uma rede inteligente de medidores eletrônicos de energia elétrica é atender à classe de medidores de BT (Baixa Tensão) e as normas que a define. Nessa classe, os recursos de hardware são limitados o que diminui as possibilidades de soluções. As fontes desses medidores conseguem prover no máximo $30 \mathrm{~mA}$ e qualquer solução implantada no medidor não poderá consumir mais do que isso. A rede deve ser capaz de funcionar mesmo sem a alimentação direta da rede elétrica, pois os nós devem enviar alarmes de falta de energia para as concessionárias atuarem de forma mais assertiva na solução do problema, atendendo às aplicações de IoT. Por isso, a alimentação por baterias é necessária quando faltar energia à rede de medidores.

Devido a utilização de baterias, é importante a aplicação de técnicas para maximizar o tempo de vida da rede. Entende-se como tempo de vida o tempo até a morte do primeiro nó. Uma das formas mais eficientes para maximizar o tempo de vida é desligar parte dos nós que não estão sendo utilizadas. Atualmente, os fabricantes de soluções para medição de energia elétrica devem manter os parâmetros e registradores de seus aparelhos por no mínimo 120 horas, quando estão sem energia e alimentados por baterias, conforme norma ABNT NBR (Norma Brasileira) 14519:2011 Medidores eletrônicos de energia elétrica (estáticos) [ABNT 2011]. Nesse caso, é necessário que o ciclo de trabalho seja o menor possível para aumentar o tempo de vida da rede.

Qualquer solução proposta para comunicação com medidores de energia deve atender a norma ABNT NBR (Norma Brasileira) 14522:2008 Intercâmbio de informações para sistemas de medições de energia elétrica. Esta norma é extremamente rígida quanto ao protocolo de comunicação utilizada pelos medidores e soluções para coleta dos dados de medição. Comandos de leitura de faturamento não podem sofrer qualquer alteração durante a transmissão e são checados durante a homologação do sistema no Inmetro. A agregação de dados, por exemplo, não é prevista e não pode ser utilizada por nenhuma solução de comunicação com os medidores [ABNT 2008].

O objetivo deste trabalho é projetar e desenvolver uma rede sem fio de medidores de energia que colete de forma automática as informações de medições de consumo e alarmes, disponibilizando em um servidor para acesso externo, atendendo as regulamentações brasileiras atuais. Além disso, a solução proposta realiza de forma dinâmica o cálculo do ciclo de trabalho de acordo com a formação e alterações na topologia, atendendo ao tempo mínimo de 120 horas de vida da rede, quando alimentada por baterias. Validações e testes realizados comprovaram o atendimento aos requisitos do setor.

O restante deste trabalho está organizado da seguinte forma: na seção 2, são apresentados os principais trabalhos relacionados à IoT aplicada as redes elétricas inteligentes. A seção 3 apresenta a descrição da solução proposta, especificando hardware, software e a técnica para cálculo dinâmico do ciclo de trabalho. A seção 4 apresenta as validações 
e resultados experimentais da pesquisa e, por último, na seção 5, são apresentadas as conclusões e trabalhos futuros.

\section{Trabalhos Relacionados}

Sustentada pela massiva instalação de sensores, a popularização das aplicações de IoT proverá informações cada vez mais precisas e reais. Na medição de energia elétrica, os primeiros trabalhos relacionando IoT e as smartgrids focaram em entender as características das duas tecnologias e as possibilidades da interação entre elas. Em [Yun and Yuxin 2010], os autores analisam as tecnologias e apontam a necessidade de uma comunicação nos dois sentidos pelas smartgrids para que os serviços atendam aos requisitos da IoT.

Em [Chen et al. 2011], os serviços e os benefícios que as smartgrids podem prover foram especificadas considerando IoT. Segundo os autores, a automação da coleta de dados de faturamento permitirá aos usuários um maior controle dos gastos com energia elétrica. Aplicações de automação residencial poderão ser incorporadas às redes elétricas inteligentes. As linhas de transmissão podem ser monitoradas em tempo real e os veículos elétricos poderão ser monitorados com maior assertividade.

A partir da definição dos serviços que podem ser oferecidos pelas smartgrids baseados em IoT, simulações e aplicações práticas começaram a ser especificadas e implementadas. Na pesquisa desenvolvida em [Ou et al. 2012], os autores apresentam os resultados do desenvolvimento prático de IoT nas linhas de transmissão de alta tensão chinesas. Instalou-se sensores de velocidade dos ventos, temperatura dos cabos de transmissão, umidade, chuvas e movimento dos cabos. Todo o sistema é alimentado por baterias solar e a transmissão das informações coletadas é realizada através de redes $3 \mathrm{G}$.

No trabalho desenvolvido em [Arif et al. 2013], os autores projetam e implementam um protótipo de medidor inteligente que utiliza GSM (Global System for Mobile Communications) e ZigBee para criar uma aplicação de smartgrids na Arábia Saudita. O medidor aproveita o amplo uso da rede GSM, com a sua capacidade de envio de SMS (Short Message Service), e da tecnologia ZigBee para enviar e coletar os dados de consumo de energia. No mesmo ano, a empresa de energia elétrica sueca Vattenfall apresentou um projeto para monitoramento em tempo real de interupções de energia e eventos de qualidade de energia, descrito em [Garpetun 2013], com o objetivo de aprimorar o atendimento a seus consumidores. Nesse trabalho, utiliza-se PLC (Power Line Communication) para comunicação com os medidores. Leituras entre 1 e 10 minutos são realizadas e, caso um medidor não responda, o sistema considera que o mesmo está sem energia elétrica.

As tecnologias de comunicação para medidores eletrônicos de energia elétrica aplicadas em IoT são apresentadas em [Arnachalam et al. 2014]. Os autores comparam as tecnologias considerando frequência utilizada, taxa de transmissão, área de cobertura, aplicações suportadas pelas tecnologias e suas limitações. A pesquisa sobre tecnologias de comunicação para medidores eletrônicos aplicadas em IoT é importante como referência para escolha em implementações práticas. $\mathrm{O}$ trabalho não define uma tecnologia mais adequada para aplicação de IoT em smartgrids. Em [Spano et al. 2014], são propostos e desenvolvidos protótipos para medidores inteligentes de energia elétrica, roteadores, servidores distribuídos para IoT e uma interface de usuário acessível por smartphones. $\mathrm{O}$ medidor inteligente proposto pelos autores não faz parte da smartgrid, ele é um módulo a 
parte que pode ser conectado às tomadas das residências. Para comunicação entre o medidor e o servidor IoT os autores utilizam um gateway ZigBee. A aplicação de smartphone acessa esses servidores IoT para coletar as informações geradas pelos medidores inteligentes.

Os trabalhos da literatura não apresentam solução para aumentar a vida útil da rede quando utiliza-se baterias para alimentação dos módulos de comunicação. Além disso, as aplicações práticas são voltadas para mercados específicos e, por isso, não atendem as normas que regem as redes elétricas inteligentes brasileiras.

\section{Arquitetura da Rede Elétrica Inteligente}

\subsection{Funcionamento}

Nesta seção, a arquitetura da rede inteligente para medição de energia elétrica é apresentada. A figura 1 apresenta a arquitetura da rede que se divide em três módulos: módulo concentrador de dados (1), módulo de comunicação sem fio (2) e módulo de medição de energia elétrica (3).

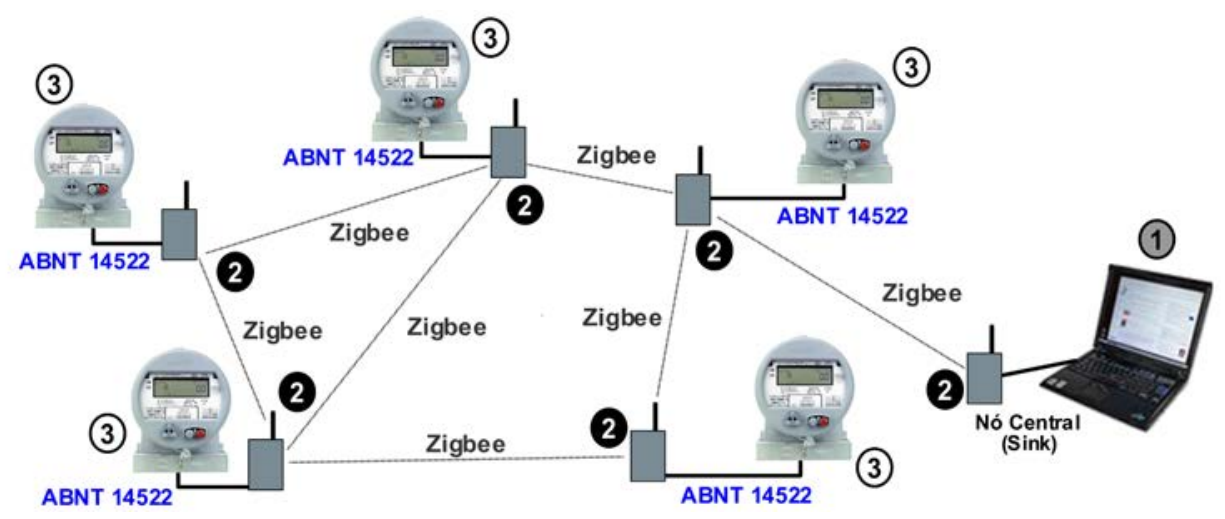

Figura 1. Rede sem fio de medidores eletrônicos de energia elétrica

O módulo concentrador de dados disponibiliza uma interface gráfica de usuário desenvolvida em LabView para controle das leituras de dados de consumo de energia elétrica dos clientes conectados à rede além de disponibilizar os dados em servidores na nuvem. A interface desenvolvida é apresentada na figura 2-(a). O número 1 indica o campo com os medidores componentes da rede. O número 2 representa os campos de configuração da porta serial. O número 3 apresenta os campos de comando e resposta.

O módulo de comunicação sem fio é responsável pelo tráfego de dados na rede, executando roteamento, controle de fluxo e criptografia dos dados. Dispositivos transceiver XBee, apresentados na figura 2-(b), foram utilizados para implementação. A árvore de coleção é formada e mantida pelo protocolo ZigBee. A prevenção de colisão é realizada através de CSMA/CA. O módulo de medição de energia elétrica é responsável pela medição dos dados de consumo dos clientes e disponibilização desses na rede. Para este módulo, utilizou-se o medidor eletrônico de energia elétrica Spectrum $\mathrm{S}$, fabricado pela empresa Nansen S/A. A figura 2-(c) ilustra esse medidor. O medidor Spectrum S utiliza o DSP (Digital Signal Processor) TMS320C203 da Texas Instruments. As implementações desenvolvidas no sistema embarcado do medidor foram realizadas utilizando o compilador Code Composer 4.12. 


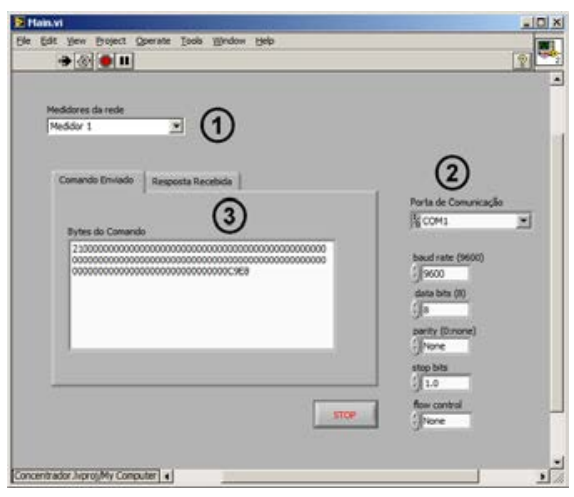

(a) Concentrador de dados

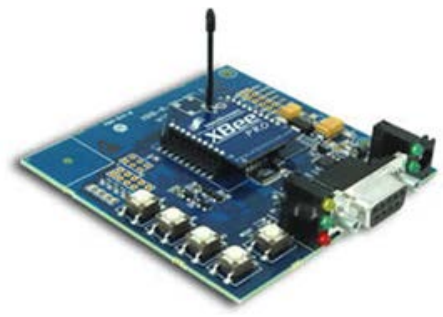

(b) Comunicação sem fio

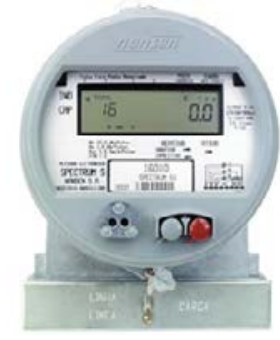

(c) Medição de energia elétrica

Figura 2. Módulos componentes da rede elétrica inteligente

Os módulos medição de energia elétrica e concentrador de dados comunicamse através do módulo de comunicação sem fio. O módulo de medição de energia envia os dados de consumo registrados e a parametrização dos mesmos para a rede. $\mathrm{O}$ módulo de comunicação sem fio roteia esses dados, passando a informação por outros nós da rede, quando necessário, até o concentrador que disponibilizará os dados. Os dados coletados pela rede são disponibilizados na internet pelo sistema MECE ( $\mathrm{Me}$ tering Center) da Siemens S/A e os consumidores podem acompanhar seu consumo diariamente. As comunicações entre os módulos de medição de energia elétrica e o módulo de comunicação sem fio são realizadas utilizando a interface de comunicação serial RS232 dos medidores eletrônicos, conforme os padrões definidos na norma ABNT 14522 [ABNT 2008]. A comunicação entre os módulos de comunicação sem fio é feita utilizando ZigBee. A figura 3 apresenta as velocidades de transmissão entre os módulos.

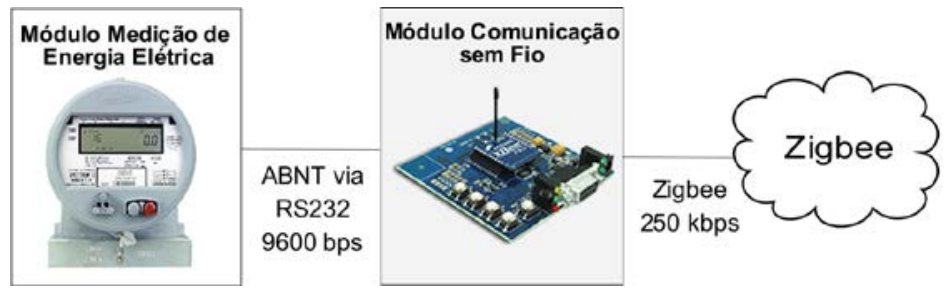

Figura 3. Velocidade de transmissão entre os módulos

Como os registradores dos medidores eletrônicos são atualizados a cada minuto e considerando a regulamentação da ANEEL que exige a atualização do mostrador de consumo do cliente sempre que o registrador de consumo do medidor for alterado, uma rede inteligente de medidores necessita de leituras dos dados de consumo e parâmetros do medidor a cada minuto. Outro fator importante que exige a leitura a cada minuto é o levantamento da curva de consumo do cliente, o que propiciará a aplicação da fatura horo-sazonal ${ }^{1}$ e um melhor dimensionamento do sistema elétrico.

\footnotetext{
${ }^{1}$ Aplicação de tarifas diferenciadas de consumo de energia elétrica e demanda de potência de acordo com a hora de utilização do dia e períodos dos anos.
} 
Quanto ao regime de operação, deve-se levar em consideração o envio de 2 blocos de 258 bytes (comandos 21 e 23 da ABNT 14522 de tamanho fixo), informando os dados de medição e configuração dos medidores, a cada minuto por nó da rede. O comando 21 informa toda a parametrização do medidor. O comando 23 contém informações sobre o consumo instantâneo, demanda máxima e contador de pulsos de energia. Através da análise conjunta desses comandos é possível verificar se o medidor está enviando o consumo zerado devido a falta de energia, falha do medidor ou alteração indevida de terceiros. Os sistemas atuais de medição possuem algoritmos implementados que relacionam falta de energia, comando 21 e comando 23 para tomar decisões e disparar ações. Para isso, é requisito das concessionárias que o medidor continue enviando o consumo durante a falta de energia. Além disso, utiliza-se o envio a cada minuto para montar a curva de consumo, aplicar tarifação horosazonal e atender norma.

\subsection{Ciclo de Trabalho Dinâmico}

Esta seção apresenta o ciclo de trabalho dinâmico, solução proposta para maximizar o tempo de vida da rede inteligente para medição de energia elétrica, considerando o processo de formação da rede e as alterações na topologia que podem acontecer durante seu funcionamento. Inicialmente, todos os nós possuem ciclo de trabalho de $100 \%$. O concentrador é parametrizado na concessionária com os nós componentes de sua rede, pois os clientes devem ser identificados para serem faturados. Assim checa-se, durante a formação, se todos os nós estão ativos. Caso um nó não entre na rede, a concessionária deverá verificar o problema e corrigí-lo, pois nenhum cliente pode ficar sem seu faturamento. Assim que todos os nós entram na rede, o primeiro ciclo de trabalho é calculado e enviado para a rede. Enquanto a rede está energizada os módulos de comunicação permanecem alimentados pela sua própria fonte e o ciclo de trabalho continua $100 \%$. A rede passa a trabalhar dentro do ciclo de trabalho calculado quando ocorre uma falta de energia e os módulos passam a ser alimentados por baterias. No início de cada minuto, os nós acordam, transmitem as informações de consumo de energia elétrica e voltam ao estado inativo. Para isso, é necessário que todos os nós estejam sincronizados, acordando no tempo exato para enviar os dados. Essa sincronização é realizada pelo relógio interno do módulo medição. Para ativar ou desativar o módulo de comunicação sem fio, o módulo de medição envia um comando via RS232 para o mesmo. Foi necessário o desenvolvimento de um sistema embarcado específico para o medidor realizar as atividades descritas.

Sempre que a rede volta a ser alimentada pele rede elétrica o primeiro serviço realizado pelo módulo concentrador é a checagem do sincronismo dos nós da rede. Para isso, ele envia comandos específicos de leitura de hora e data para os módulos de medição de energia. Se necessário, um novo ciclo de trabalho é calculado considerando as diferenças de sincronismo. Medidores Spectrm S possuem relógio com precisão de $30 \mathrm{PPM} /$ ano. Se a diferença for maior que a precisão esse medidor provavelmente está com problemas e um alarme é gerado no concentrador. Após esse alarme a concessionária pode realizar maiores checagens para entender o motivo da diferença e verificar se os medidores estão com problemas técnicos ou estão sendo alterados de forma indevida por terceiros.

A metodologia para cálculo do ciclo de trabalho deve ser aplicável a todas as configurações possíveis para redes de medidores de energia elétrica. A comunicação é realizada entre os módulos de comunicação e o módulo concentrador de dados. Como exemplo, considere uma rede de medidores formada de acordo com a topologia ilustrada 
na figura 4. Para cada nó da rede, um número de saltos $(d)$ até o concentrador de dados (sink) está apresentado. Essa profundidade é identificada durante a formação da rede.

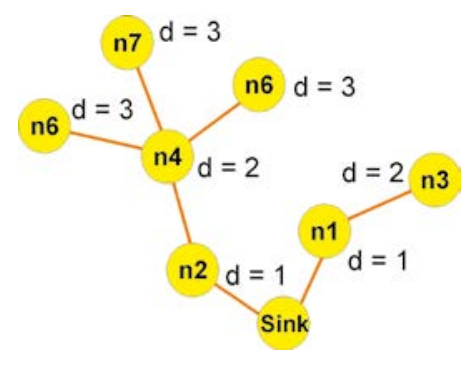

Figura 4. Exemplo de rotas entre nós

Quanto ao mecanismo de gestão dinâmica do ciclo de trabalho, sempre que um novo nó entra na rede um comando em difusão é enviado pelo mesmo, avisando a todos de sua entrada. Após receber o comando, o módulo concentrador calcula o novo ciclo de trabalho e envia o valor em difusão para todos da rede. $\mathrm{O}$ valor é assumido pelos nós e passa a ser o novo ciclo de trabalho da rede.

Para cálculo do valor do ciclo de trabalho, é necessário conhecer o tempo total necessário para que todos os nós da rede possam enviar seus dados. Neste trabalho, considera-se apenas o tempo de transmissão para o cálculo do tempo total de envio das informações. O tempo total necessário para enviar os dados é diretamente proporcional à quantidade de nós da rede $(N)$, a profundidade de cada nó $\left(d_{i}\right)$ e ao tempo de transmissão em um enlace $(t)$. O tempo de transmissão em um enlace está relacionado ao tamanho do pacote e à velocidade de transmissão. O tamanho do pacote de dados é definido de acordo com a quantidade de dados que devem ser lidos de um nó da rede, sendo essa quantidade igual para todos os nós em uma rede para medição de energia elétrica.

Para que todas as informações cheguem até o concentrador, serão necessárias $e$ transmissões, onde $e=\sum_{i=1}^{N} d_{i}$. Considerando que transmissões simultâneas não acontecem e também que todas as transmissões são bem sucedidas, o tempo total para transmissão seria: $T=t \times e$. Assim o ciclo de trabalho será suficente para atender o número máximo de 40 nós da rede.

Entretanto, colisões e erros podem acontecer durante as transmissões. Considere $p$ a probabilidade de sucesso de uma transmissão e $Z$ uma variável aleatória com distribuição geométrica que representa o número de tentativas para envio com sucesso de um pacote em um determinado enlace, tem-se: $Z \sim G e o(p)$. O valor esperado da variável aleatória $Z$ é: $E(Z)=\frac{1}{p}$. Sendo assim, no pior caso, o tempo total necessário para enviar todos os pacotes será definido por: $T=\frac{1}{p} \times t \times e$

Portanto, na solução proposta, no início de cada minuto, os nós ficam acordados durante $T$ segundos para transmitirem as informações de consumo de energia elétrica e voltam ao estado inativo. O ciclo de trabalho deve ser atualizado sempre que um novo nó entrar na rede ou uma manutenção de rota for executada. Para realização da manutenção da rota, todos os nós continuam informando a sua profundidade durante o funcionamento, essa informação é registrada no concentrador e sempre checada. Caso ocorra uma alteração nesse valor, causado pela mudança de rotas, derivada de bloqueios ou mudança na qualidade do link, um novo ciclo de trabalho é calculado. Após realizar o 
cálculo do novo valor de $T$, este deve ser atualizado e enviado para todos os nós da rede que passarão a utilizar o novo valor. O responsável pela execução do cálculo do ciclo de trabalho é o módulo concentrador de dados.

\section{Validações e Resultados Experimentais}

Esta seção apresenta as validações e resultados experimentais do sistema proposto neste trabalho. Os experimentos dividem-se em quatro grupos: medição do consumo de energia dos modos de funcionamento (seção 4.1), probabilidade de sucesso em cada transmissão (seção 4.2), tempo de vida da rede (seção 4.3) e o percentual de sucesso no recebimento das informações de consumo (seção 4.4). Todos os experimentos foram realizados na empresa Nansen S/A utilizando a plataforma XBee Série 2 - XB24BPDK juntamente com o amperímetro Minipa ET-1110 DMM para medição de consumo dos nós. Em todos os experimentos, utilizou-se a bateria Duracell Alcalina de 9V padrão 6LR61 de $580 \mathrm{mAH}$ como fonte de alimentação para os módulos de comunicação sem fio.

\subsection{Medição do Consumo de Energia dos Modos de Funcionamento}

O primeiro experimento foi realizado com o intuito de verificar o consumo de energia nos modos de funcionamento e o tempo de vida de um nó da rede em cada modo. Para este experimento, os nós 4 e 5 , apresentados na figura 6-(b) foram utilizados. Um nó responsável pela transmissão e outro pela recepção. A distância entre os nós não foi alterada pois o ZigBee não possui controle de potência [Farahani 2008].

Para a coleta dos dados de consumo de energia, um circuito foi montado entre o módulo comunicação sem fio e sua alimentação. O circuito consiste em inserir um amperímetro em sequência com a bateria para medir o consumo da mesma. A cada cinco minutos, registrou-se o consumo do módulo de comunicação. A figura 5 ilustra o sistema montado e utilizado para medição das informações. Percebe-se na foto o módulo de medição de energia elétrica (1), medidor Spectrum S, o módulo comunicação sem fio ZigBee (2) e o amperímetro registrando o consumo (3).

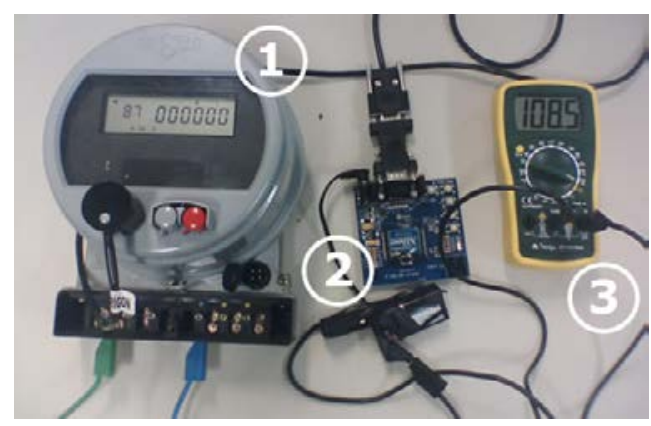

Figura 5. Registro de consumo do módulo de comunicação sem fio

Os experimentos foram realizados durante o período de 165 minutos, contabilizando 33 registros. Três modos de operação do módulo XBee foram avaliados: inativo, recepção e transmissão. No modo inativo, o rádio encontra-se desligado de forma que apenas a placa consome energia. O consumo do modo de funcionamento inativo é de aproximadamente 4,5 mA, pois é necessário manter os leds de funcionamento e a porta de comunicação RS232 para ativar ou destivar o módulo. Para execução do experimento 
de medição do consumo durante a recepção de dados, o módulo comunicação sem fio permanece, durante todo o tempo, recebendo dados. Nesse modo, o consumo permanece constante na faixa de $87 \mathrm{~mA}$. Durante a transmissão, o consumo aumenta consideravelmente se comparado com os modos anteriormente avaliados. O consumo para transmissão na placa de desenvolvimento permaneceu próximo a $110 \mathrm{~mA}$.

A tabela 1 mostra o tempo de vida de um nó considerando seu modo de operação. O tempo de vida de um nó que permanece no modo inativo durante todo o período é $580 \mathrm{mAH}$ (energia fornecida pela bateria) / 4,5 mA (consumo do modo de funcionamento), o que resulta em 128 horas e 53 minutos. Assim calcula-se o tempo de vida do nó para os demais modos de funcionamento.

Tabela 1. Tempo de vida de um nó

\begin{tabular}{|c|c|}
\hline Modos de Operação & Tempo \\
\hline \hline Inativo & 128 horas e 53 minutos \\
\hline Recepção (Rx) & 6 horas e 44 minutos \\
\hline Transmissão (Tx) & 5 horas e 16 minutos \\
\hline
\end{tabular}

A corrente necessária para transmissão do módulo de comunicação, $110 \mathrm{~mA}$, é superior a fornecida pela fonte do medidor de energia elétrica que é $30 \mathrm{~mA}$. Sendo assim, é impossível alimentar o módulo de comunicação com a fonte do medidor.

\subsection{Probabilidade de Sucesso em cada Transmissão}

O objetivo desse experimento é encontrar o valor da probabilidade de sucesso em cada transmissão $(p)$ definido na seção 3.2. A figura 6-(a) ilustra a rede implementada, que utiliza a topologia mesh. Escolheu-se os nós 11 e 5 para esse experimento devido a maior quantidade de saltos para comunicação. O módulo de comunicação sem fio foi configurado para realizar apenas uma tentativa de envio do pacote pela rede. Caso ocorra algum problema durante o envio, não é realizada uma nova tentativa de transmissão. Além disso, o módulo permanece com o rádio ligado durante todo o experimento. Esse experimento foi realizado durante o intervalo de 5 horas, tempo suficiente para obter o valor de $(p)$. A cada 5 segundos, o nó 11 envia os dados de consumo e configuração. Ao final, totalizouse o envio de 7.198 comandos pelo nó 11 dos quais 7.133 foram recebidos pelo nó 5 . Sendo assim, o percentual de sucesso em cada transmissão foi $p=0,991$. Esse valor será utilizado nas próximas seções para o cálculo do valor do ciclo de trabalho.

\subsection{Tempo de Vida da Rede}

O objetivo dessa seção é avaliar o tempo de vida da rede para pilha de protocolos ZigBee e pilha de protocolos ZigBee com o ciclo de trabalho dinâmico proposto neste trabalho. Em todos os experimentos, a cada 60 segundos, o módulo de medição de energia envia os dados de consumo e configuração através do módulo de comunicação sem fio.

Para execução dos experimentos uma mesma tabela de roteamento foi utilizada. A figura 6-(b) ilustra as rotas definidas. Escolheu-se o nó 7 para medir o tempo de vida da rede devido a sua maior quantidade de filhos na rede, o que acarreta em um número maior de envio e recepção de dados. Os dados foram coletadas a cada 30 minutos, através da montagem especificada na figura 5. O tempo de vida da rede, para tecnologia ZigBee 


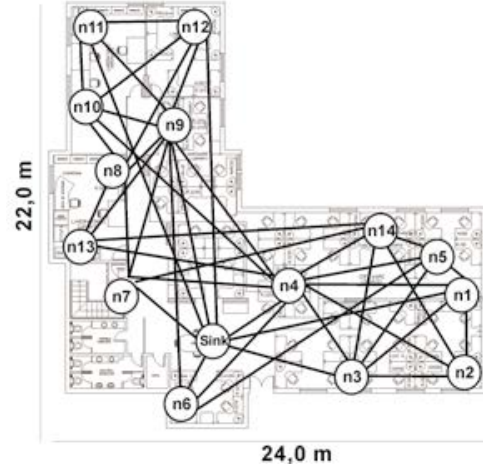

(a) Topologia da rede

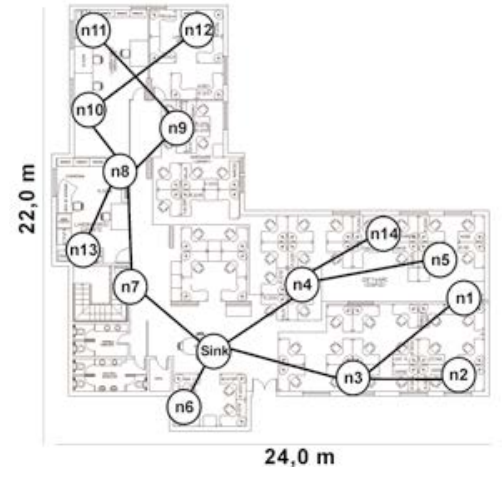

(b) Rotas definidas entre nós

Figura 6. Topologia e Rotas

original, foi de 433 minutos ou 7 horas e 13 minutos. Um tempo de vida pequeno, mas dentro das expectativas devido às medições realizadas na seção 4.1.

Baseando-se na topologia apresentada na figura 6- $(a)$ e na proposta para maximizar o tempo de vida da rede, calcula-se o valor do ciclo de trabalho. Considerando a velocidade de transmissão de $250 \mathrm{kbps}$ do ZigBee, 516 bytes transmitidos, o valor do tempo $(t)$ para cada transmissão realizada por enlace da rede será 0,016 segundos. Conforme apresentado na seção 3, o número total de transmissões realizadas na rede $(e)$, considerando o pior caso, no qual não acontece transmissão simultânea entre os nós, é um somatório da profundidade de todos os nós da rede. Considerando a topologia apresentada na figura 6, o valor de $e$ é igual a 31. De acordo com o experimento realizado na seção 4.2, a probabilidade de sucesso em cada transmissão é de $p=0,991$. O tempo total necessário para enviar todos os pacotes será: $T=\frac{1}{p} \times t \times e=\frac{1}{0,991} \times 0,016 \times 31=0,50$ segundos.

Ao tempo $\mathrm{T}$ calculado é necessário acrescentar o tempo gasto para comunicação entre o módulo de medição de energia elétrica e o módulo de comunicação sem fio, pois conforme especificado na seção 3.1, a porta de comunicação RS232 dos medidores possui velocidade de 9600 bps. A comunicação entre os dois módulos acontece de forma simultânea em toda a rede, então basta acrescentar o tempo necessário para uma transmissão dos 516 bytes entre o módulos. De acordo com os dados apresentados, o valor de $T$ será: $T=0,50+\left(\frac{516 \times 8}{9600}\right)=0,93$ segundos.

Como cada nó deverá permanecer ligado durante 0,93 segundos em cada 60 segundos, conclui-se que o ciclo de trabalho para a rede analisada é de 1,55\%. Estudos mostram que o valor de ciclo de trabalho deve ser próximos a $1 \%$ para que redes de sensores sejam funcionalmente viáveis [Guo et al. 2009].

O gráfico da figura 7 apresenta a comparação entre os tempos de vida das duas tecnologias analisadas, percebe-se que ao atingir $4,8 \mathrm{~V}$ o nó da rede para de comunicar, pois a plataforma XBee Série 2 - XB24BPDK necessita de uma alimentação de no mínimo $4,8 \mathrm{~V}$ para o funcionamento de seus componentes. Observa-se também que o mecanismo para maximizar o tempo de vida da rede aumenta o tempo de vida em torno de 17 vezes. O tempo máximo de vida da rede será 126 horas e 30 minutos para o ZigBee com ciclo 
de trabalho dinâmico, atendendo ao requisito da norma ABNT 14519 [ABNT 2011].

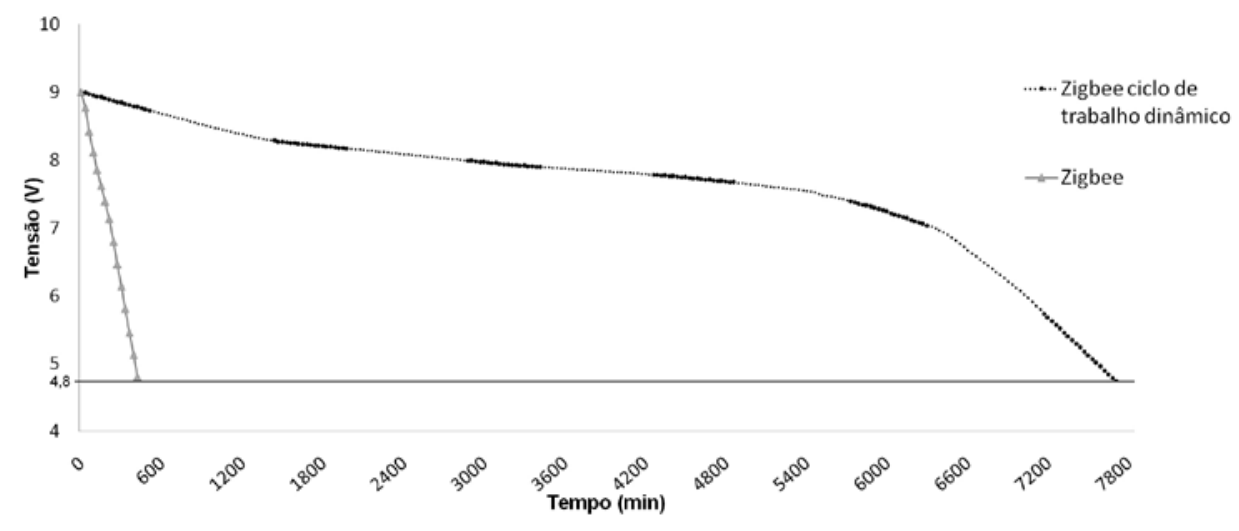

Figura 7. Tempo de vida das tecnologias analisadas

\subsection{Percentual de Sucesso no Recebimento das Informações de Consumo}

Para cálculo do percentual de sucesso no recebimento de informações de consumo utilizou-se a mesma topologia de rede dos experimentos de tempo de vida para o ZigBee com ciclo de trabalho dinâmico, ilustrada na figura 6-(b). Compara-se os dados recebidos pelo módulo concentrador de dados com os dados registrados nos módulos de medição de energia elétrica. Esse experimento foi realizado durante 8 horas, tempo maior que o utilizado no experimento da (seção 4.2), pois neste os envios são a cada minuto.

Os comandos trafegados na rede são compostos por 258 bytes. Os últimos dois bytes são utilizados para informar o CRC (Cyclic Redundancy Check) de 16 bits que é calculado sobre os demais 256 bytes. O módulo concentrador só considera um comando recebido como válido se o calculo de seu CRC estiver correto.

Após as 8 horas de testes, o módulo de medição de energia elétrica interrompe o envio de comandos e o registro do envio dos mesmos. Em cada módulo de medição de energia elétrica da rede, registrou-se o envio de 958 comandos. Ao todo, 13.412 comandos foram enviados pela rede. O módulo concentrador de dados registrou o recebimento de 13.239 comandos corretos. Assim tem-se um percentual de sucesso de $98,7 \%$ no recebimento das informações.

\section{Conclusões e Trabalhos Futuros}

O trabalho apresentou uma aplicação prática para IoT nas redes elétricas inteligentes brasileiras utilizando as normas vigentes. A rede desenvolvida possibilitará um maior controle de gastos pelos clientes das concessionárias de energia elétrica devido ao acesso facilitado e em tempo real de consumo através de computadores na nuvem.

Importante ressaltar que, assim como as tecnologias, as normas brasileiras precisam avançar. Os protocolos definidos na norma ABNT 14522 são extremamente ultrapassados e rígidos. Propostas simples para diminuir o tráfego de dados na rede podem ser implementadas sem perda de confiabilidade. Agregação de dados e pacotes com tamanho variável, sem a obrigatoriedade de 258 bytes fixos, são exemplos.

Foi apresentada também uma metodologia para cálculo do ciclo de trabalho dinâmico cujo objetivo foi manter um ciclo de trabalho mínimo para leitura dos dados 
de consumo, mesmo ocorrendo alterações na topologia da rede. A principal contribuição foi o sensível aumento do tempo de vida da rede. Com a utilização do ciclo de trabalho dinâmico, a rede passou a atender as normas vigentes. O ciclo de trabalho manteve-se dentro dos padrões definidos pela literatura, próximo à $1 \%$. Além disso, a rede proposta mostrou-se eficiente quanto ao consumo máximo de um conjunto de medição definido nas normas vigentes para medição de energia elétrica. Considerando o percentual de sucesso nas transmissões realizadas, a ciclo de trabalho dinâmico mostrou-se eficiente atingindo um percentual de $98,7 \%$, o que comprova a confiabilidade do sistema proposto.

Como trabalho futuro, pretende-se considerar, durante o cálculo do tempo necessário para enviar todos os pacotes, as transmissões simultâneas que poderão acontecer nos diversos enlaces da rede. Assim, o tempo necessário para enviar todos os pacotes pode ser diminuído. Outro trabalho futuro seria calcular o $p$ dinamicamente. Esse valor seria utilizado no cálculo do ciclo de trabalho de forma que a solução proposta fosse adaptável às condições do meio de transmissão sem fio.

\section{Referências}

ABNT (2008). NBR 14522: Intercâmbio de informações para sistemas de medição de energia elétrica Padronização. ASSOCIAÇÃO BRASILEIRA DE NORMAS TÉCNICAS ABNT, Rio de janeiro.

ABNT (2011). NBR 14519: Medidores eletrônicos de energia elétrica (estáticos) - Especificação. ASSOCIAÇÃO BRASILEIRA DE NORMAS TÉCNICAS ABNT, Rio de Janeiro.

Arif, A., Al-Hussain, M., Al-Mutairi, N., Al-Ammar, E., Khan, Y., and Malik, N. (2013). Experimental study and design of smart energy meter for the smart grid. In Renewable and Sustainable Energy Conference (IRSEC), 2013 International, pages 515 - 520, Ouarzazate. IEEE.

Arnachalam, V., Jain, S., Kumar, N., Paventhan, A., Chinnaiyan, V., and Pradish, M. (2014). Survey on smart grid technologies- smart metering, iot and ems. In Electrical, Electronics and Computer Science (SCEECS), 2014 IEEE Students' Conference on, pages 1 - 6, Bhopal. IEEE.

Bello, O. and Zeadally, S. (2014). Intelligent device-to-device communication in the internet of things. Systems Journal, IEEE (Issue: 99 ), PP:1 - 11.

Chen, X., Liu, J., Li, X., Sun, L., and Zhen, Y. (2011). Integration of iot with smart grid. In Communication Technology and Application (ICCTA 2011), IET International Conference on, pages 723 - 726, Beijing. IET - IEEE.

Farahani, S. (2008). Zigbee Wireless Networks and Transceivers. Elsevier, Oxford, UK.

Garpetun, L. (2013). Smart meters in operation center. In Electricity Distribution (CIRED 2013), 22nd International Conference and Exhibition on, pages 1 - 3, Stockholm. IET - IEEE.

Guo, S., Gu, Y., Jiang, B., and He, T. (2009). Opportunistic flooding in low-duty-cycle wireless sensor networks with unreliable links. In MobiCom '09: Proceedings of the 15th annual international conference on Mobile computing and networking, pages 133-144, New York, NY, USA. ACM.

Ou, Q., Zhen, Y., Li, X., Zhang, Y., and Zeng, L. (2012). Application of internet of things in smart grid power transmission. In Mobile, Ubiquitous, and Intelligent Computing (MUSIC), 2012 Third FTRA International Conference on, pages 96 - 100, Vancouver, BC. IEEE.

Spano, E., Niccolini, L., Pascoli, S., and Iannaccone, G. (2014). Last-meter smart grid embedded in an internet-of-things platform. Smart Grid, IEEE Transactions on (Volume:PP, Issue: 99 ), PP:1.

Yun, M. and Yuxin, B. (2010). Research on the architecture and key technology of internet of things (lot) applied on smart grid. In Advances in Energy Engineering (ICAEE), 2010 International Conference on, pages $69-72$, Beijing. IEEE. 